\title{
Fuzziness, Cognition and Cybernetics: an outlook on future
}

\author{
Enric Trillas ${ }^{1}$ Settimo Termini ${ }^{1,2}$ Marco Elio Tabacchi $^{2,3}$ Rudolf Seising $^{1,4}$ \\ ${ }^{1}$ European Center for Soft Computing, Mieres (Asturias), Spain \\ ${ }^{2}$ Dipartimento di Matematica e Informatica, Università di Palermo (Italy) \\ ${ }^{3}$ Istituto Nazionale di Ricerche Demopolis (Italy) \\ ${ }^{4}$ Friedrich-Schiller-Universität, Jena (Germany)
}

\begin{abstract}
In the present paper, we connect some old reflections about the relationships existing between the theory of fuzzy sets and cybernetics with modern, contemporary analyses of the crucial (better: unavoidable) role that fuzziness plays in the attempts at scientifically describing aspects of information sciences. The connection, which has a basic conceptual origin, has been triggered also by the recent 50th anniversary of Norbert Wiener' death which has been instrumental in looking again at some crucial aspects of the birth of information sciences in the midst of last Century. Fuzzy sets are an essential part of this revolution and share all the innovations as well as the difficulties of this towering scientific enterprise which has changed the vision of what a scientific approach must be when dealing with something like information so different from the old matter and energy. These considerations are helpful in looking in an enlarged way at how treat and consider the notion of cognition.
\end{abstract}

Keywords: cybernetics, fuzzy set, fuzziness

\section{Introduction}

The historical notions discussed in [1] are in our opinion important and crucial for present day research. Let us be more clear, what is really crucial in many new fields (for instance in the attempts at grasping meaningful aspects of human cognition or natural language) is not the possibility of introducing techniques developed in some chapters of mathematics or logic or engineering in itself. This can be done routinely now for obtaining very specific results. However, in many cases this is done, mechanically also in ways and modalities which do not increment the level of our understanding of the considered problems. Since many people - correctly in our view - think that imprecision is in many places unavoidable (Without error and imprecision we perhaps could not have seen life itself. Rita Levi-Montalcini, the Nobel prize biologist, entitled her autobiography "Praise of imperfection") what is crucial is to give space to an informal notion of "unsharpness" [2]. This should be the more consonant possible with the characterizing features of the various problems. So what is needed is not - for some inexplicable reasons - fuzziness as it is formalized in the theory of fuzzy sets. Fuzziness (and the theory of fuzzy sets) is crucial in our view, in this specific moment of the historical evolution of these theories, since it is the form in which the formalization of the informal notion of vagueness (or of "unsharpness", in general) presents itself less conditioned by specific assumptions and at the same time it also allows for the use of "linguistic" quantities in the same formalism, providing in this way really a bridge between hard and human sciences. Let us also remember that, recently, it has been observed that the presence of network makes all these considerations extremely more cogent than before. (These has been taken as paradigmatic also in a recent paper appeared in Scientific American [3].)

\subsection{Structure of the paper}

The structure of the paper is as follows: In Section 2 the main reasons for looking at fuzzy sets in a innovative way will be briefly presented and in Section 3 the connection that we can today establish between the old cybernetic motivations and what today could be called "cognitive" instances associated to very contemporary issues like the ones provided by networks will be outlined. Conclusions cannot but be provisional, but we strongly believe that these kinds of analyses can help us in doing real progresses without doing again in their entirety the mistakes done in past decades.

\section{How to look anew at fuzzy sets}

In this Section we shall provide a rough survey of a new way of looking at fuzzy sets which could help in using the same theory in new domains.

\subsection{Fuzzy sets, imprecision and non-random uncertainty}

It can be said that as it was originally introduced by Zadeh [4], fuzzy set theory mainly deals with two important linguistic phenomena, imprecision and non-random uncertainty, and that fuzzy sets can be applied, among others, to the study of dynamical systems described by sets of imprecise linguistic rules, and to the random uncertainty associated to some linguistic statements [5]. For instance, the theory of possibility can manage with non-random uncertainty, fuzzy control with dynamical systems which behavior can be described linguistically, and fuzzy probability with random fuzzy events. The 
ground of fuzzy set theory lies in the, historically not surprising, fact that predicates acting in a universe of discourse generate linguistic collectives in it; collectives [6], except when they degenerate in just a single classical set, are cloudy linguistic entities neither well known, nor easy to specify 'thought' entities whose appearances, or states, are just membership functions, fuzzy sets allowing to see their projections inside the fog of ordinary language. Hence, fuzzy sets can be seen as a starting point for the currently non existing scientific study of linguistic collectives. In sum and grossly speaking, fuzzy sets deal with ordinary language; they are mathematical entities contextually reflecting collectives, and that are mathematically modelled by their membership functions. They meant to pass from an old world of exact thinking represented by sets, to a new world of inexact thinking represented by them; the future of fuzzy sets can be seen around a new mathematical study of ordinary, or common sense, reasoning in which the central idea is, instead of 'deducing' from precise premises reflecting totally known information even if not fully describing something, that of 'conjecturing' [7] from imprecise premises reflecting information partially known and also able to reach creative conclusions, that is, to increase the informative content of the premises or previous information. Fuzzy sets have to do with both the representation of information, and to obtain a new one by just a 'previous thinking' as it is done always in searching for a new aspect of a problem, and that, latter on, should be either formalized, or checked against some reality to acquire the status of 'new' knowledge. Of course, in these processes of conjecturing are included those of deducing, abducing, and also lucubrating [8]. Without no doubt, it can be also said that fuzzy sets' idea was born in the 'cultural' neighborhood of cybernetics, where analogical computers [9] were seriously taken into account. Fuzzy sets are indeed analogical entities in front of the digital crisp sets and, since most of the human knowledge is essentially analogical, it is not at all surprising that fuzzy sets can be suitable for representing, at least, expert knowledge. In fact, the first application of fuzzy sets to the control of machines, introduced in 1972 by the late Abe Mamdani [10], can be considered as a method for the management of imprecise expert knowledge, and who knows if, in a future, and provided analogical quantum computers [11] were actually constructed, fuzzy sets will not play some role in their functioning. If from a philosophical and scientific point of view fuzzy sets are but measures, from a technological one they are just analogical tool constructs representing knowledge.

\subsection{Fuzzy sets and the meaning of their linguistic label}

For a lot of time since 1965, the year Zadeh published his seminal paper 'Fuzzy Sets' [4], the mathematical nature of fuzzy sets in relation with the meaning of their linguistic label, was not clearly explained. They were simply viewed as membership functions generalizing the characteristic function of crisp sets and, supposedly, representing its meaning in the universe of discourse, but without counting with a meaning's operational definition [12]. If philosophers largely debated on the meaning of 'meaning', they don't attended the representation of meaning, it lacked of a scientific study that can be considered it started with the work of Zadeh, and in a form close to the Wittgenstein of the 'Philosophical Investigations', when he states that almost always 'the meaning of a word is its use in language'. How can be mathematically described, even if not defined, the use or management in language of a linguistic label?

If $P$ is a linguistic label, or predicate, acting in a universe of discourse $X$ through the elemental statements " $x$ is $P$ ", for a suitably management of $P$ the two binary relations in $X$, that empirically come from linguistic perception, from its use,

- $x=_{P} y \Leftrightarrow x$ shows the property named $P$ equally than $y$ shows it $\Leftrightarrow x$ is equally $P$ than $y$

- $x \leq_{P} y \Leftrightarrow x$ is less $P$ than $y$,

should be known [5, 6].

When both relations coincide, it is said that the use of $P$ in $X$ is precise, rigid, or crisp, $=_{P}$ is an equivalence, and $X$ is partitioned in the equivalence classes in the quotient set $X /=,[x]=\left\{y \in X ; y={ }_{P} x\right\}$. When $\leq_{P} \neq==_{P}$ that, provided it can be supposed $=_{P}=\leq_{P} \cap \leq_{P}^{-1}$, implies that it is not $\leq_{P} \subseteq \leq_{P}^{-1}$, it is said that the use of $P$ in $X$ is imprecise, flexible, or fuzzy. In any case, the graph $\left(X, \leq_{P}\right)$ represents the qualitative, or primary, meaning of $P$ in $X$. In this way, the previously amorphous universe of discourse $X$, is softly structured thanks to the use of $P$ in it. The simple and usual act of 'talking' on a property recognizable in the elements of $X$, endows $X$ with the arcs of this graph; an idea corresponding with the intuitive one that rational speech introduces some kind of 'ordering' in the universe of discourse, also corresponding to the establishment of some necessary link between ordering and understanding. Nevertheless, the graph does not exhaust the 'meaning' of $P$ in $X$, and when it is $\leq_{P}=\emptyset$, it can be said that $P$ is metaphysically used in $X$, that $P$ is metaphysical in $X$. Notice that it is thanks to the relation _ $P$ that can be seen the variability of the property named $P$ along the elements of $X$; that it is $\leq_{P}=/=_{P}$, is what permits to say that the use of $P$ is imprecise in $X$. If $P$ is not metaphysically used in $X$, that is, if $\leq_{P} \neq \emptyset$, then a measure of the extent of $P$ in $X$, is a mapping $\mu_{P}: X \Rightarrow[0,1]$, such that,

$$
\begin{aligned}
& \text { 1. } x \leq_{P} y \Rightarrow \mu_{P}(x) \leq \mu_{P}(y) \\
& \text { 2. } z \text { maximal for } \leq_{P} \Rightarrow \mu_{P}(z)=1 \\
& \text { 3. } z \text { minimal for } \leq_{P} \Rightarrow \mu_{P}(z)=0 .
\end{aligned}
$$

Once the graph $\left(X, \leq_{P}\right)$ is known it can be said that $P$ is measurable in $X$, and once a measure $\mu_{P}$ is known that it is effectively measurable in $X$ [6]. The three former properties are not sufficient, in general, to specify 
a measure of which it only exists a single one if the predicate is precise; to specify a measure either more information on the use of $P$, or to establish a hypothesis on it, is necessary. In any case, each measure $\mu_{P}$ is the membership function of the fuzzy set in $X$ labeled $P$. Fuzzy sets are defined by measures of the extent up to which the elements in $X$ are $P$, show the property named $P$; shortly speaking it can be said that fuzzy sets are measures of meaning, like probabilities are measures of random uncertainty, and fuzzy entropies are measures of fuzziness. It should be noticed that each quantity $\left(X, \leq_{P}, \mu_{P}\right)$ represents a good enough knowledge on the meaning of $P$ in $X$ for its scientific consideration; it can be said that such quantities are the typically scientific domestication of meaning [13], and can offer a new perspective for studying both fuzzy sets and fuzzy logic. It should be noticed that if the use of $P$ in $X$ is rigid, it is $x={ }_{P} y \Leftrightarrow x<_{P} y \& y<_{P} x \Rightarrow=A_{P}(x)=A_{P}(y)$, and hence, $A_{P}$ is constant in the classes modulo $P$, the only values $A_{P}$ can take are 0 or $1, A_{P}^{-1}(1)$ is the crisp subset specified by $P$ in $X, A_{P}^{-1}(0)$ its classical complement, and one of them can be empty.

\subsection{How a fuzzy set is designed "in the praxis"}

In the praxis, a fuzzy set is designed by means of the information on its linguistic label that is available and that, most of the times, is not the full relation $\leq_{P}$, but a part of it; there are cases in which obtaining $\leq_{P}$ can be very difficult. Hence and very often, neither it is always $\leq_{P}$ completely known, nor it can be stated that the designed membership function $\mu_{P}^{*}$ is truly a measure, but some unknown approximation of it. Consequently, the designer cannot work with $\leq_{P}$ but only with the total order defined by $x \leq_{\mu^{*} P} y \Leftrightarrow \mu_{P}^{*}(x) \leq \mu_{P}^{*}(y)$, called the working meaning of $P$ in $X$, that, provided $\mu_{P}^{*}$ were actually a measure or, at least, it can be supposed it verifies property (1), and since then, $x \leq_{P} y \Rightarrow$ $\mu_{P}^{*}(x) \leq \mu_{P}^{*}(y) \Leftrightarrow x \leq_{\mu_{P}^{*}} y$, implies $\leq_{P} \subseteq \leq_{\mu_{P}^{*}}$, that is, the working meaning extends the qualitative meaning of $P$. The act of measuring $P$, modifies its qualitative meaning by adding more arcs to it [6]. Notice that since in most cases the relation $\leq_{P}$ has not total, or linear, character, cannot coincide with the linear orders $\leq_{\mu P}$. When there is coincidence, it is said that the measure perfectly reflects the qualitative meaning of $P$.

In those cases in which the measure does not perfectly reflect the qualitative meaning, and since in science is not at all rare to manage measures with complex values, it could be suitable to substitute the real interval $[0,1]$ by the complex one $\{a+b i ; a, b \in[0,1]\}$, the complex circle, endowed with the usual partial or$\operatorname{der} a_{1}+b_{1} i \leq a_{2}+b_{2} i \Leftrightarrow a_{1} \leq a_{2} \& b_{1} \leq b_{2}$, and with analogous properties [6] to the former (1), (2), and (3). This substitution cannot guarantee that a complex-valued measure will perfectly reflect the qualitative meaning, but just that can offer more possibilities for it, since the working order will be not linear. This is a substitution that can be equivalently seen at taking an interval-valued measure by just changing the view of $a+b i$ by the interval $[a, b]$, that corresponds to a par- ticular type of the so-called type-2 fuzzy sets reflecting that the value of the measure carries with the uncertainty coming from only being sure that it is in the interval $[a, b]$. Analogously, and instead of the real or the complex unit intervals, it can be taken the set $[0,1]^{[0,1]}$, of the fuzzy sets in the unit interval (type-2 fuzzy sets) that contains images isomorphic to both the unit interval and the complex unit interval, for those cases in which the only that can be asserted is that the value of the measure is, for instance, either 'around 0.7', or 'high' [14]. In this form, all the types of fuzzy sets currently considered, are integrated thanks to the quantities representing the meaning of its linguistic label.

The full meaning of a linguistic label $P$ is not unique, but it is actually context-dependent and purpose-driven. Each quantity $\left(X, \leq_{P}, \mu_{P}\right)$, real, complex or fuzzy valued, is obtained through what the designer can know, in a given context, of the use, action or behavior of $P$ in $X$, or through some reasonable hypothesis he could be able to make on such behavior. This last is the often considered case in most applications, in which the realvalued measure, the membership function, is supposed to be trapezoidal, or just triangular.

\subsection{A fuzzy set as a 'measure of the meaning'}

Once seen that the membership functions of fuzzy sets mean nothing else than a 'measure of the meaning' of its linguistic label, it can be remembered the famous words of Lord Kelvin shortened to 'If you cannot measure it, it is not science'. There are, notwithstanding and at least, two aspects introducing important differences between Lord Kelvin's times and ours. In the first place, it is the fact that if, let's say, that science is essentially concerned with matter and energy, fuzzy set theory is with knowledge and information, and related with the so called Information Technologies. In a second place, that in Lord Kelvin's science there were and are, known systematic procedures and laboratory methods, to measure the basic parameters of the studied things, but now and for what concerns, for instance, the design of membership functions, the situation is different and more linked to some analogy with virtual objects, than with physically real objects. It is not the same to study the chemical composition of an organic product, or the movement of a star, than to study the meaning of a written piece, or the control of a machine whose behavior is known by the knowledge of the experts in their functioning once linguistically described. Anyway, the evolution of fuzzy logic towards Zadeh's Computing with Words and Perceptions, soon or latter will conduct towards the mathematical representation of statements larger and more complex than the more or less simple rules considered in control [15], and this will mean to face the necessity of considering different ways of expressing conditional statements, and the linguistic connectives 'and', 'or', 'not', etc., already known since there is not a universal form of expressing them in language, like it is in classical logic and set theory, but respectively represented in fuzzy logic by residuated implications, S-implications, conjunctive implications, t- 
norms, t-conorms, negation functions, etc. If of all that there are a lot of mathematical models, if fuzzy logic has a remarkable armamentarium for the representation of statements, and for doing deductive inferences with them, what is not yet clear enough is to which linguistic subjects such armamentarium is applicable and to which is not. For instance, fuzzy logic only considers functionally expressible connectives, but no suitable criteria are known for recognizing this hypothesis in concrete cases. In sum, it seems that fuzzy logic is approaching the time in which it should face a turning point. The great subjects fuzzy logic deals with are linguistic imprecision and non-random uncertainty, not to say anything of the very important but scientifically pending subjects of ambiguity, the presence of multiple meanings, and common sense non-deductive reasoning [8] with imprecise, non-randomly uncertain, and ambiguous words. The only proper way to properly affront it is, in the author's view, the transformation of fuzzy logic in a kind of 'physics' of imprecision, non-random uncertainty and ambiguity, in a new experimental science that, based in Natural Language, can count with mathematical models able to give important parameters to be experimentally measured and found in the same study of language and not by abstract mathematical thought considerations; to transform the study of language from a logic and humanistic one in a scientific one. When fuzzy logic was initially developed in the past Century's seventies and eighties, the almost only back referents for its study were classical and multiple-valued logics, but now it should be centered in Natural Language.

If current fuzzy logic already meant an important progress in the way asked by John von Neumann of introducing mathematical analysis in the study of those subjects without a just 'yes' or 'not' hypothesis for its validity, it can be the right moment to go an step ahead turning towards the AI's 'gordian knot' of trying to mechanically thinking like people usually do.

\section{Fuzziness, cognition (and the network)}

If fuzziness has to become a new "physics of the immaterial" [16], rooted more in human imperfection, ambiguity and vagueness than in the frigidity of numbers, then a major push in this direction should come from a new relationship with Cognitive Sciences. It can be said that a certain form of diffidence exists between the two communities, and this despite a continuous cross-fertilization, involving especially the Soft Computing scientists and cognitive psychologists, as evidenced by a growing number of special sessions and cross-discussions in conferences from the two disciplines. Part of this mistrust can probably be traced back to the origins of fuzziness and Soft Computing [17], fields of research stemming mainly from Electrical Engineering, and as such considered by many in the human sciences overly complicated and less approachable than alternatives such as the ones offered by e.g. expert systems. The alternating fortunes of Artificial Intelligence - another discipline involved by the multidisci- plinary vocation of Cybernetics - had not helped either, as a decades long lack of substantial results alienated the sympathy of the sectors of cognitive sciences more interested in behaviour, and pushed them towards neurosciences, which in the nineties started to gain popularity. Another push to reconnect human sciences and disciplines of imprecision (namely fuzzy logic, neurocomputing, evolutionary computing, probabilistic computing, chaotic computing and machine learning) was made at the start of the present century, when Lotfi Zadeh stated than Soft Computing was "a coalition of methodologies which are drawn together by a quest for accommodation with the pervasive imprecision of the real world" [18].

\subsection{Why fuzziness is crucial in cognition...}

It was already noted by Zadeh in 1970 [19] that a paradox exists involving the ways of human reasoning and computer problem solving. It was clear then, as it unfortunately is now, that while computing power is still increasing (though not necessarily forever [20]), some of the most basic tasks carried out by humans are still out of grasp for machines. True, in the last years a number of research projects which are examples of spectacular failures in the eighties are finally starting to ripe: the first commercial applications of autonomous driving, speech recognition, and - albeit to a lesser extent of expert knowledge systems [21] give a strong impression that intelligence in machines is now not as distant as it was just ten years ago. Such applications' apparent successes hide the fact that most of the result are obtained through more computing power, better sensors and huge data repositories. In such achievements there often is not an ounce more of intelligence in the proper sense than it was yesterday (it is the triumph of big data, not of big brains), and the reality check in this sense often comes from the lack of flexibility demonstrated at the minimum changes in external conditions, as in the case of a recent autonomous drive test, in which, after a first day of success, the second day was completely disrupted by a slight change in the weather conditions. Such occurrences, which are more and more common in AI's practical applications, demonstrate that there still is a paradigm shift needed to happen: veritable intelligence, in the human sense, has to come from a different approach to the complexity in real word. Examples of such kind of behaviour are commonplace, and especially poignant every time a typical human activity - exactly the ones that Cognitive Science studies and that are so difficult to replicate in machines - becomes the center of interest. There is a common thread between technology and human sciences [22, 23] and the disicplines ad the edge are fuzziness and cognition.

\section{2. .... and what the network adds}

While it may seem an hyperbole at first, on reflection it may be just right to say that we live in a networked word. The availability of a pervasive, cheap and readily implementable network for information exchange, 
coupled with the commercial and social success of mobile platforms and the affordability of smartphones and other networkable devices means that unless we leave for a very remote island or desert, we are constantly immersed in a flow of information with no diaeresis. For Cognitive Science this development has determined a huge shift of interest toward the social components of information, and for the first time it has been possible to devise and execute a wealth of experiments dealing with exchange of information and the social mechanisms underlying it - technology allowed quantity and quality of information that is a far cry from the pioneering experiments of Milgram [24]. A huge push in this direction comes from the discovery by Barabasi and collaborators [25] of a specific kind of networks, called small words, known for a certain ubiquity in natural and man made self-organization. Small World topology can explain a system evolution in simple terms, related to the ability of the element to connect and disconnect from each other, and such simple rules can be found in a lot of different man-made and natural occurring networks, such as the skyways, many biological systems and even in networks that are more conceptual in nature, such as the topology of concept building in human thinking [26]. The study of topologies, be they human interactions in social networks or complex interplay in living cells has exacerbated the curse of dimension: as vaste amounts of data are more and more available, any hope for absolute precision is lost, despite all the added computational and storage power. Soft Computing techniques, especially evolutionary and probabilistic computing, as well as intelligent data analysis, have been a great source of alleviation for the curse, but this is a good time to reconsider how the likes of fuzziness, uncertainty and computing with words [27] are a fitting proposal when dealing with networks; the view of Fuzzy Sets as an experimental science $[16,28]$ offers the tools needed for the trade, and especially an ecologic, transparent process of analysis. In what can be defined as network thinking [3], data should come with its own semantics, and a natural way of dealing with such coupling can be through the use of methodologies that are respectful of the inherent imprecision in human artifacts and natural occurrences. As such, a future in which networks are more and more important and present in our reality spells wonders for Fuzzy Sets Theory and for all the many facets of fuzziness.

\subsection{Fuzziness, thinking and cognition}

And we are back to Cybernetics - and not by chance: there is a strong connection, outlined in the previous sections, between the first serious approach to the study of systems, be they natural or artificial, and the future of Cognitive Sciences.

\section{Looking for the future}

From the historical analysis done in [1] and in this contribution, it seems interesting to envisage what some future developments could be if a few of the analyses done in the previous pages should turn to be right and correct. First. We should try to merge all the results done in FST (and Soft Computing) with the other traditions of scientific investigation without having the obsession to follow the same methodologies considered standard in the various communities. The only paradigm to which we must pay a tribute is the one of scientific rigour. We should try to preserve as long as possible all the freshness of the new ideas. And preserving freshness means that we should fix also the agenda of the new crucial questions and not uncritically accept the agenda of other nearby disciplines. Second. We should recognize that in information sciences the relationships between conceptual notions and formalisms are different and less linear than in other disciplines. To look at the past history of Cybernetics can help us in following promising paths without doing too many mistakes (as in the last case has happened) Third. We should look for the most promising fields for innovative applications, and that means that we must do a sort of experimental work in looking for innovative extensions of our ideas in new fields.

\section{Acknowledgements}

The work of one of the authors (ET) has been partially funded by the Foundation for the Advancement of Soft Computing (Mieres, Spain), and by the Spanish Government's project MICIIN/TIN 2011-29827-C02-01.

\section{References}

[1] Rudolf Seising, Marco Elio Tabacchi, Settimo Termini, and Enric Trillas. Fuzziness, cognition and cybernetics: an historical perspective. In in this volume, 2015.

[2] Rita Levi-Montalcini. In praise of imperfection: my life and work. Alfred P. Sloan Foundation series, New York: Basic Books, I c1988, 1, 1988.

[3] John Edward Terrell, Termeh Shafie, and Mark Golitko. How networks are revolutionizing scientific (and maybe human) thought. Scientific American Guest Blog (available online), 2014.

[4] Lotfi A Zadeh. Fuzzy sets. Information and control, 8(3):338-353, 1965.

[5] Enric Trillas and Luka Eciolaza. Fuzzy Logic: An Introductory Course for Engineering Students, volume 320. Springer, 2015.

[6] Enric Trillas, Settimo Termini, and Claudio Moraga. A naïve way of looking at fuzzy sets. Fuzzy Sets and Systems, forthcoming, 2015.

[7] Enric Trillas. A model for "crisp reasoning" with fuzzy sets. International Journal of Intelligent Systems, 27(10):859-872, 2012.

[8] Enric Trillas. En defensa del razonamiento creativo. Universidad Pública de Navarra, 2014.

[9] L Basañez, N Batle, G Ferraté, J Grané, and E Trillas. A first mathematical approach to $\sigma$-transform. Journal of Mathematical Analysis and Applications, 92(1):224-233, 1983. 
[10] Ebrahim H Mamdani and Sedrak Assilian. An experiment in linguistic synthesis with a fuzzy logic controller. International journal of man-machine studies, 7(1):1-13, 1975.

[11] C P Williams and S H Clearwater. Explorations in Quantum Computing. Springer, NY, 1998.

[12] Hung T Nguyen, Nadipuram R Prasad, Carol L Walker, and Elbert A Walker. A first course in fuzzy and neural control. CRC press, 2002.

[13] Enric Trillas. How does science domesticate concepts? Archives for the Philosophy and History of Soft Computing, (1), 2014.

[14] Settimo Termini. On some vagaries of vagueness and information. Annals of Mathematics and Artificial Intelligence, 35(1-4):343-355, 2002.

[15] Enric Trillas and Sergio Guadarrama. Fuzzy representations need a careful design. International Journal of General Systems, 39(3):329-346, 2010.

[16] Marco Elio Tabacchi and Settimo Termini. Theory of computation, fuzziness and a physics of the immaterial. In Davide Ciucci, Javier Montero, and Gabriella Pasi, editors, Proceedings of the 8th conference of the European Society for Fuzzy Logic and Technology, Advances in Intelligent Systems Research. European Society for Fuzzy Logic and Technology, Atlantis Press, 2013.

[17] Rudolf Seising and Marco Elio Tabacchi. A very brief history of soft computing. In W Pedrycz and M Reformat, editors, 2013 Joint IFSA World Congress NAFIPS Annual Meeting. IEEE SMC, 2013.

[18] Lotfi A Zadeh. Foreword. Applied soft computing, 1(1):1-2, 2001.

[19] L. A. Zadeh. Fuzzy languages and their relation to human and machine intelligence, man and computer. In Proceedings of the International Conference, Bourdeaux, 1970.

[20] Marco Elio Tabacchi. Salvi ed al sicuro: Singolarità di vinge, crescita tecnologica, limiti di processo. In A Auricchio, M Cruciani, M Rega, and M Villani, editors, Scienze Cognitive: paradigmi sull'uomo e la tecnologia. Atti del X convegno annuale AISC, volume 2 of Giornale italiano di Neuroscienze, Psicologia e Riabilitazione, pages 213218. Nea Science, 2013.

[21] Fabio Aurelio Dasaro, Valerio Perticone, and Marco Elio Tabacchi. L'obiezione di una lady ed il computer che vince ai telequiz. come la flessibilità ha consentito all'intelligenza artificiale di superare un limite immaginario. In Ines Giunta, editor, FlessibilMENTE - Un modello sistemico di approccio al tema della flessibilità, La Società Formativa, pages 379-398. Pensa Multimedia, 2014.

[22] Fabio D'Asaro, Valerio Perticone, Marco Elio Tabacchi, and Settimo Termini. Reflections on technology and human sciences: rediscovering a common thread through the analysis of a few epistemological features of fuzziness. Archives for Phylosophy and History of Soft Computing, 1, 2013.
[23] Fabio Aurelio Dasaro, Valerio Perticone, Marco Elio Tabacchi, and Settimo Termini. Technology and human sciences: a dialogue to be constructed or a common tread to be rediscovered? In W Pedrycz and M Reformat, editors, 2013 Joint IFSA World Congress NAFIPS Annual Meeting. IEEE SMC, 2013.

[24] Duncan J Watts. Six degrees: The science of a connected age. WW Norton \& Company, 2004.

[25] A L Barabási. Linked: The New Science of Networks. Basic Books, 2002.

[26] Maria Petrou, Marco Elio Tabacchi, and Roberta Piroddi. Networks of concepts and ideas. The Computer Journal, 53(10):1738-1751, 2010.

[27] L.A. Zadeh. Fuzzy logic = computing with words. Fuzzy Systems, IEEE Transactions on, 4(2):103 111, 1996.

[28] Marco Elio Tabacchi and Settimo Termini. Some reflections on fuzzy set theory as an experimental science. In Anne Laurent, Olivier Strauss, Bernadette Bouchon-Meunier, and Ronald R. Yager, editors, Information Processing and Management of Uncertainty in Knowledge-Based Systems, volume 442 of Communications in Computer and Information Science, pages 546-555. Springer International Publishing, 2014. 